\title{
SILICON MICROMACHINED MASS FILTER FOR A LOW POWER, LOW COST QUADRUPOLE MASS SPECTROMETER
}

\author{
J. J. Tunstall ${ }^{1}$, S. Taylor ${ }^{1}$, R. R. A. Syms ${ }^{2}$, T. Tate ${ }^{2}$, and M. M. Ahmad ${ }^{2}$ \\ ${ }^{1}$ Department of Electrical Engineering and Electronics, Liverpool University, Brownlow Hill, \\ Liverpool L69 3GJ, United Kingdom \\ ${ }^{2}$ Optical and Semiconductor Device Section, Department of Electronic and Electrical Engineering, \\ Imperial College of Science, Technology and Medicine, London SW7 2AZ, United Kingdom
}

\begin{abstract}
This article considers the design, realisation and initial tests of a Quadrupole Mass Spectrometer with a novel silicon micromachined mass filter. The construction of this device is discussed along with its mounting on a conventional ion source and testing in a vacuum system. Results are presented for the operation of this mass lens including a mass spectrum, and the effect of cage voltage, emission current and pressure on the performance.
\end{abstract}

\section{INTRODUCTION}

Quadrupole Mass Spectrometers (QMS) have been widely researched and developed as sensors and diagnostic tools for over 40 years. They have found a wide range of applications in the medical field, chemical process industries and more recently in process monitoring in semiconductor fabrication plants where ultra clean processes for ULSI are a priority.

A QMS consists of three components: (i) ion source , (ii) mass filter and (iii) detector [1]. The conventional arrangement uses circular metallic rods as the mass filter (typical dimensions: rod-length: $50-225 \mathrm{~mm}$; diameter: 5-15 $\mathrm{mm}$ ) excited electrically. We have produced a micro-machined quadrupole lens made from silicon with chromium sputtered $\mathrm{SiO}_{2}$ optical fibre rods as the electrodes [2,3]. The metallised fibre rods of the quadrupole mass filter are mounted in pairs on two silicon substrates, which are held apart by two additional insulating cylindrical rods as shown in fig. 1.

The correct electrode spacing and alignment are achieved through the use of V-shaped grooves etched into the silicon. The silicon substrate carries only one alignment rod which mates with a groove on the other substrate. This arrangement effectively eliminates all possible degrees of freedom except axial motion. This sub-assembly was mounted onto a conventional ion source with a Faraday plate collector as detector to measure the ion current.
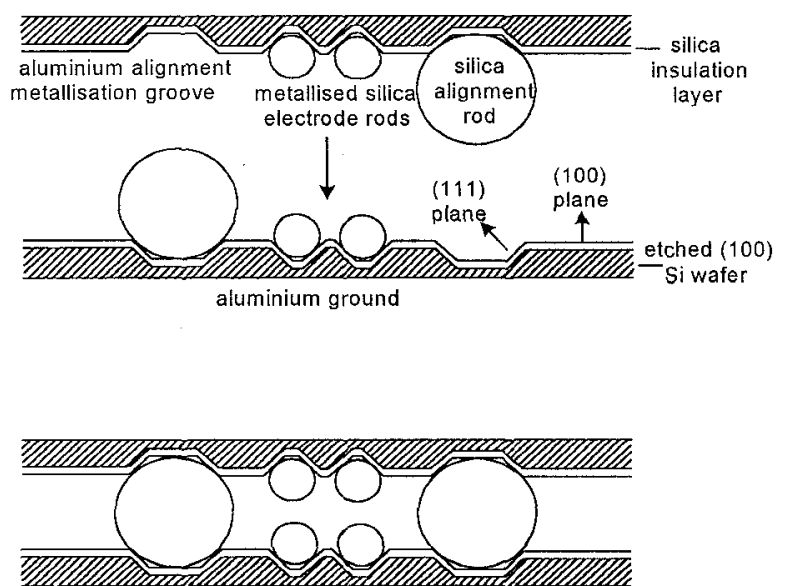

Fig. 1 Self - aligning microengineered quadrupole lens assembly

\section{THEORETICAL CONSIDERATIONS}

An ideal quadrupole mass filter consists of four electrodes with hyperbolic cross-section aligned in parallel with a potential applied to the rods of $\pm \phi_{0} / 2$. An illustration of this is given in fig. $2 \mathrm{a}$. This structure gives a hyperbolic field with a form of $\phi(x, y)=\phi_{0}\left(x^{2}\right.$ $\left.y^{2}\right) / 2 r_{0}{ }^{2}$ where $r_{0}$ is a characteristic radius. If $\phi_{0}$ is given by a time varying field of $\phi_{0}=U-V \cos (2 \pi f t)$ then a mass dependent focusing effect takes place within the quadrupole lens. Now if $U$ and $V$ are varied [4] such that the ratio of $U$ to $V$ remains constant a mass spectrum can be achieved through the transmitted ion current. In practice it is difficult to produce hyperbolic shaped electrodes so cylindrical rods are used instead. These will approximate the hyperbolic field [5] provided the electrode radius $r_{e}$ is related to the inscribed radius of the four rods $r_{0}$, shown in fig. $2 b$, by the ratio $r_{e}=1.148 r_{0}$. 
QMS based on cylindrical rods for the mass filter are now highly developed and successful [6]. Only in recent years have economic methods of precision lens assembly been devised [7]. However the mass filters are still bulky, delicate and require large drive voltages at $\mathbf{r f}$ frequencies. If the cost, the size and voltages be reduced and along with an increase of robustness then the range of applications for these types of mass spectrometer would increase.
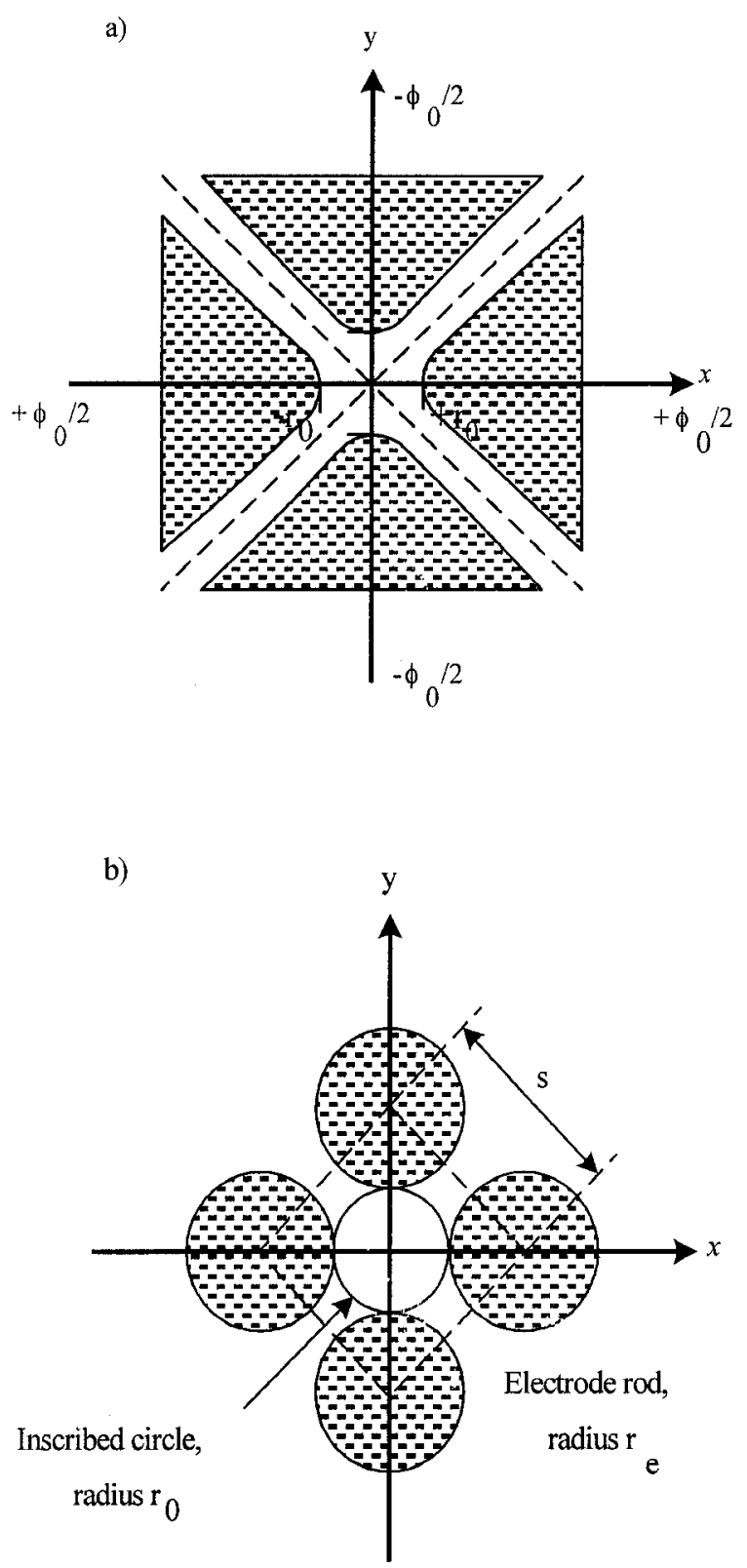

Fig. 2 Ideal electrostatic quadrupole lens consisting of a set of four parallel electrodes with hyperbolic crosssection.

a) Exact quadrupole electrode geometry.

b) Approximate quadrupole electrode geometry
Changing the size of the mass lens would effect the performance of the QMS. It can be shown that a measure of the resolution $\Delta M$ (in a.m.u.) of an electrostatic quadrupole lens is given by [6]:

$$
\Delta M \approx 3.854 \times 10^{9} V_{z} / f^{2} L^{2}
$$

where $V_{z}$ is the axial energy of an ion measured in $\mathrm{eV}$ and $L$ the electrode length. To minimise $A M, V_{z}$ should be minimised. However $V_{z}$ can not be reduced indefinitely. The product $f L$ could be increased. If the mass filter is to be reduced in size the frequency $f$ must increase. With the smallest allowable $V_{z} \approx 100 \mathrm{kT}$, (2eV), for $\Delta M=1$ a.m.u. then $f=4 \mathrm{MHz}$ a quadrupole rod length of $2 \mathrm{~cm}$ is achievable.

The maximum mass measurable is given by:

$$
M_{\max }=6.92 \times 10^{6} V_{\max } / f^{2} r_{0}^{2}
$$

For a given $V_{\max }$ and product fro, $M_{\max }$ can be determined. If the frequency is taken as above i.e. $f=$ $4 \mathrm{MHz}$ then regardless of the scale reduction with $M_{\max } \approx$ 100 a.m.u. and $V_{\max } \approx 11 \mathrm{~V}$ then $r_{0}=217.5 \mu \mathrm{m}$ which corresponds to an electrode diameter of $500 \mu \mathrm{m}$.

By scaling down the size of the quadrupole mass lens we can still achieve modest performance, with moderate $\mathrm{rf}$ frequencies and relatively low voltages. Conventional assembly methods for the mass filter would be illadvised both in terms of accuracy and cost. Here we consider a microengineering approach developed for optical fibre connectors $[2,8]$. The quadrupole body is constructed from anisotropic etched silicon. The electrodes are formed from metallised silica rods. A description of the assembly of such a device is given in the introduction [9].

\section{DESCRIPTION OF THE MICRO-MACHINING}

The grooves required for the quadrupole mass filter construction were formed by anisotropic etching of 3 in (100) orientated p-type silicon in ethylene diamine pyrocatechol (EDP). The substrate was insulated by a 1 $\mu \mathrm{m}$ thick layer of thermally grown $\mathrm{SiO}_{2}$. The electrode contacts were produced from evaporation of $\mathrm{Al}$ metal through a shadow-masked system. Windows were opened in the rear-side silica layer and an Al layer was deposited to make a ground connection.

The quadrupole rods were made from commercially available silica fibres with a diameter of $500 \mu \mathrm{m}$. They were coated with a 1000 A layer of $\mathrm{Cr}$ metal. The electrodes have been mounted so the surface of the substrate passed through the rod's centre-line. 
Obviously the micro-machined substrate will effect the equipotentials of the mass filter. In turn this will alter the ideal rod separation for the hyperbolic approximation. For the first prototype design the conventional electrode separation,(ratio of $r_{e}$ to $r_{0}$ ), will remain unchanged. A diameter of $1150 \mu \mathrm{m}$ for the alignment rod was chosen, firstly to minimise the maximum groove depth required and secondly so there would he sufficient mechanical strength of the wafer, which had an initial thickness of $400 \mu \mathrm{m}$. The rods were bonded under pressure to the substrate at $180^{\circ} \mathrm{C}$ using indium as an adhesive.

Each silicon wafer contains eight lenses of varying lengths up to $3 \mathrm{~cm}$. Yields of the order of $75 \%$ are routinely achieved. Fig. 3 shows half-lens dies of 1 and $2.0 \mathrm{~cm}$ length after fabrication and fig. 4 shows an end on view of an assembled lens. The tolerances of the initial prototypes are estimated between $1-3 \%$ and are limited by the failure to seat fully the rods during bonding.

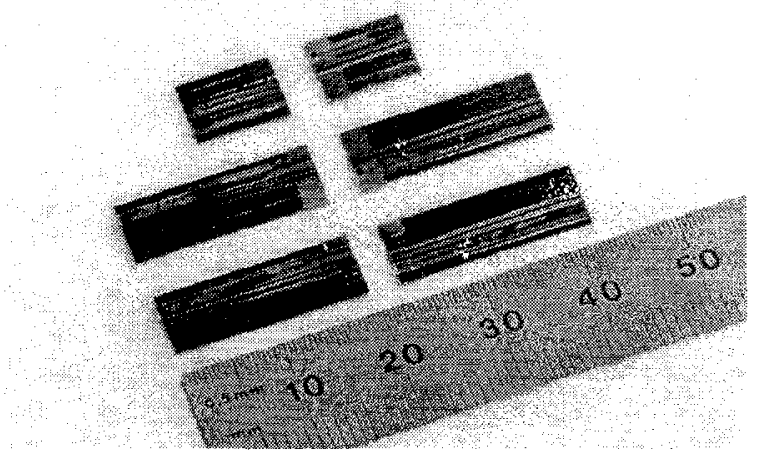

Fig. 3 This photograph shows half-lens dies of 1.0 and $2.0 \mathrm{~cm}$ length after fabrication and before assembly of the full lens unit.

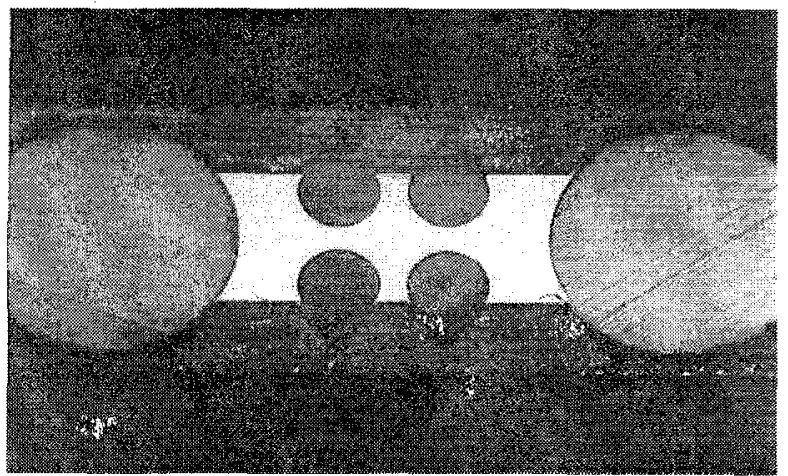

Fig. 4 This photograph shows an end on view of the assembled micromachined quadrupole mass filter

\section{DESCRIPTION OF THE TEST ASSEMBLY AND EXPERIMENTAL PROCEDURE}

The quadrupole mass filter was mounted onto a conventional VG ANAVAC ion source which was in turn attached to a vacuum flange. External connections from the mass lens and the ion source were made to pins on the outside of the flange. The ion producing source was connected to an emission regulator. The filament which produces the electrons which ionise the gas was taken at $-78.5 \mathrm{~V}$, (filament return was set at $-76.0 \mathrm{~V}$ ), the focusing element was set at $-7.7 \mathrm{~V}$ and the source cage taken at $11.0 \mathrm{~V}$. With argon gas inputted into the vacuum system argon ions were produced in the ion source which in turn passed through the mass filter. A split phase rf alternating voltage,(amplitude V), and a direct voltage, $(\mathrm{U})$, was applied to the quadrupole rods, the exact form of which is given in the section on Theoretical Considerations. The $U$ and $V$ ratio was kept fixed and the voltages scanned from high to low values. The ratio was adjusted so that the effective scan-line would pass close to the tip of the Mathieu stability diagram for singly charged argon ions.

\section{RESULTS OF THE RESOLUTION OPTIMISATION}

There are several parameters that can be varied which effect the resolution of a mass spectrometer. For our particular QMS pressure of the gas inputted, the cage voltage and emission current can be varied to maximise resolution. A typical mass spectrum is given in fig. 5 .

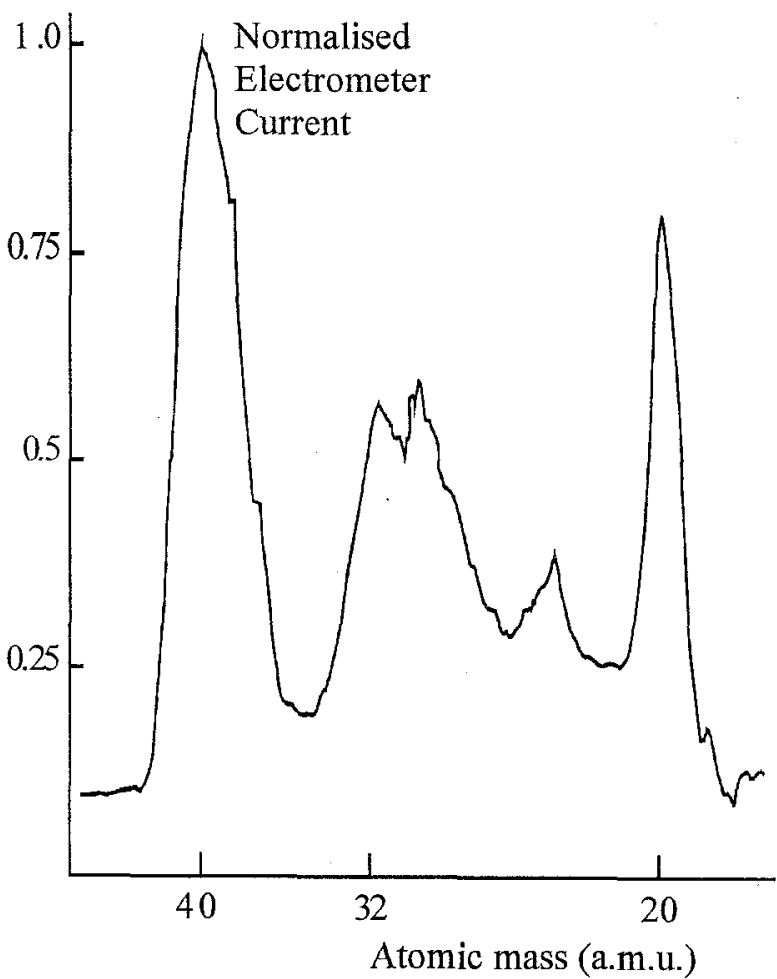

Fig. 5 This plot shows a spectrum obtained from the output of the electrometer for an argon / air gas mixture. The gas pressure was $5.0 \times 10^{-5}$ Torr and the frequency of the rf voltage was $6 \mathrm{MHz}$. The argon 40 peak is clearly seen along with a broad peak for air. The doubly charged argon peak at mass 20 is also seen. 
The plot shows the output from the electrometer for argon/air gas mixture. The gas pressure is $5 \times 10^{-5}$ Torr and the frequency of the rf voltage is $6 \mathrm{MHz}$. From the mass 40 and the mass 20 peaks the mass scale can be calibrated. Once the mass scale is known the resolution of the peaks at half height can be calculated.

Our first investigation was to see how resolution of the micro-inachined quadrupole varied with cage voltage. The spectrum was scanned for a range of cage voltages ensuring that both the singly charged argon peak and the doubly charged argon peak were recorded. Along with the positions of the peaks the electrometer current was measured so a level of sensitivity of the mass 40 and mass 20 peaks could be gauged. A general trend of decreasing resolution for increased cage voltage was seen for the singly charged argon peak. The resolution appeared to peak at cage voltage of $6 \mathrm{~V}$. As the cage voltage increased sensitivity increased also. As cage voltage became larger the ions produced became more energetic allowing more to pass through the mass filter. The more energy the ions have the less effected they are by the electric fields of the mass lens. As sensitivity increases resolution decreases with a broadening of the transmission peak. Fig. 6 shows resolution versus cage voltage.

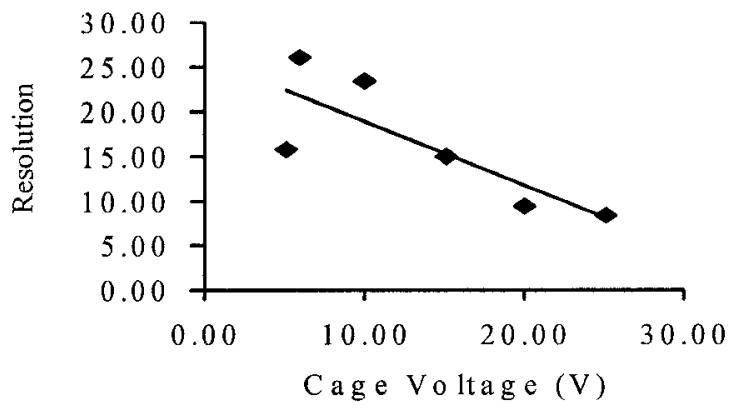

Fig. 6 This figure shows the experimental behaviour of half-peak height resolution versus cage voltage, for emission current set at $100 \mu \mathrm{A}$. As cage voltage increases resolution decreases. A best straight-line fit has been drawn through the data.

Our next investigation was to vary emission current and see its effect on resolution of the mass 40-argon peak. The cage voltage was set at $10 \mathrm{~V}$. Resolution appeared to peak at an emission current of $60 \mu \mathrm{A}$. There appears to be a decrease in resolution as emission current increases. There are three distinct regions of the curve, emission current less than $90 \mu \mathrm{A}$, emission current between $90 \mu \mathrm{A}$ and $190 \mu \mathrm{A}$ and emission current greater than $190 \mu \mathrm{A}$. These can be seen quite clearly on fig. 7 , which shows resolution versus emission current.

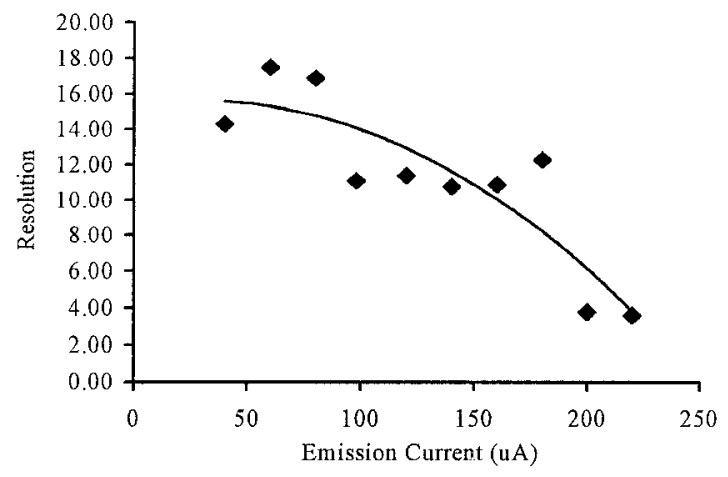

Fig. 7 This figure shows resolution versus emission current. The cage voltage was set at $10 \mathrm{~V}$. A decrease in resolution is clearly seen for increasing emission current. A best-fit curve is shown drawn through the experimental data.

Our next consideration was to see how resolution varied with pressure. The cage voltage was set at $10 \mathrm{~V}$ and the emission current was set at $80 \mu \mathrm{A}$. Resolution initially decreased for increasing pressure but then increased again. There appears to be no distinct pattern, fig. 8 bears this out.

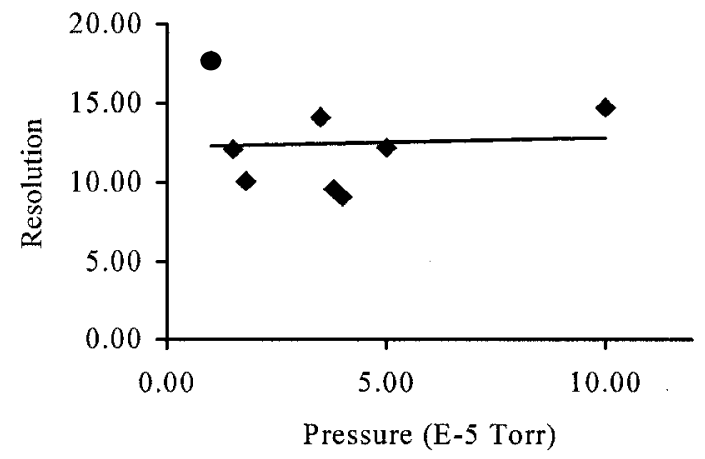

Fig. 8 This plot shows resolution versus pressure, for a cage voltage of $10 \mathrm{~V}$ and a emission current set at $80 \mu \mathrm{A}$.

An instructive plot to consider is sensitivity versus the reciprocal of the resolution, (which is proportional to $\Delta \mathrm{M})$. Fig. 9 shows a ' $\mathrm{C}$ ' type curve indicating a maximum in the resolution. There are some points that do not lie on this curve. The plot is typical of the sensitivity versus $\Delta \mathrm{M}$ for a conventional quadrupole mass filter. However the sensitivity range is not as large as for a conventional mass lens. The final curve is fig. 10, which shows sensitivity versus emission current. For low emission it shows approximately linear operation, sensitivity rising with emission current. For larger values a non-linear response occurs. This result is typical for a conventional QMS also. 


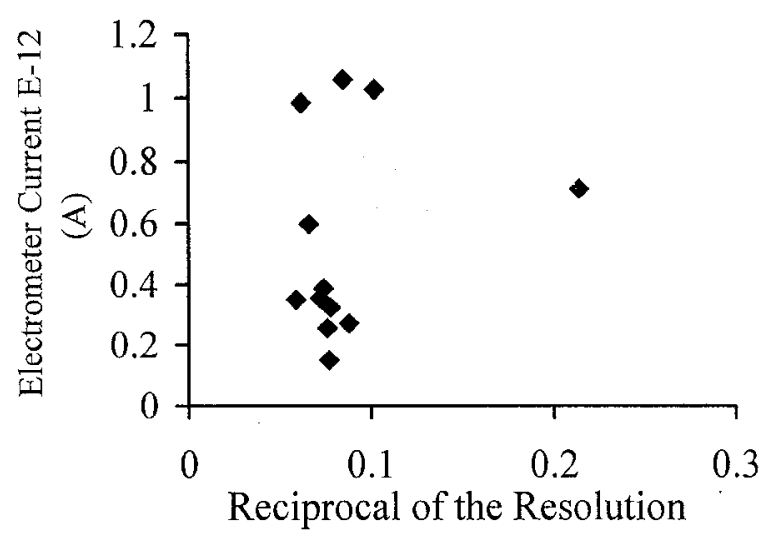

Fig. 9 The figure shows sensitivity versus the reciprocal of resolution. Emission current was set at $80 \mu \mathrm{A}$ and the cage voltage was set at $10 \mathrm{~V}$.

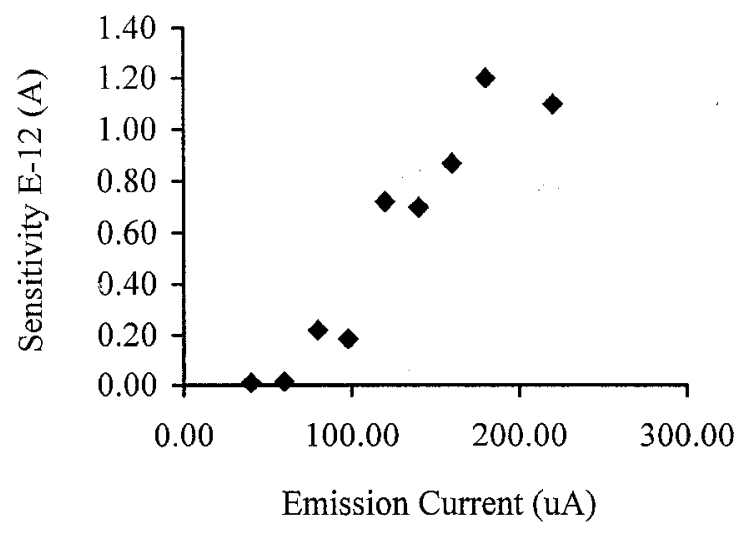

Fig. 10 The figure shows the sensitivity versus emission current. For low emission the experimental data is approximately linear but for larger values of the emission a non-linear response occurs

\section{CONCLUSIONS}

A QMS with a silicon based, fully micromachined mass filter has been designed, constructed and tested. The micromachined quadrupole mass filter behaves in some ways like a conventional mass lens. The typical sensitivity versus $\Delta \mathrm{M}$ curve was reproduced for this instrument. The resolution versus cage voltage behaves as expected, with a decrease in resolution as the energy of the argon ions is increased. Resolution versus emission current shows again as more energetic argon ions are produced the wider the mass peak, the lower the resolution. The interesting curve of resolution versus gas pressure shows little correlation. The results show definite quadrupole operation even though the conditions for optimum operation have yet to be established.

\section{ACKNOWLEDGEMENTS}

The authors would like to thank Prof. J.H. Leck and Dr. J. Batey for many useful discussions and EPSRC for their support.

\section{REFERENCES}

[1] Dawson, P.H.: 'Quadrupole mass spectrometry and its applications' (Elsevier Scientific Publishing Co., Amsterdam, 1976)

[2] Taylor, S., Syms, R.R.A., Dorey, H.A., and Tate, T.J.,: 'Improvements in and relating to quadrupole mass spectrometers'. British Patent 9,506,972, 1995

[3] Denison, D.R.: 'Operating parameters of a quadrupole in a grounded cylindrical housing', $J$. Vac. Sci. Tech., 1971, 8, pp.266-269

[4] Batey, J.H.: 'Quadrupole gas analysers (residual gas analysis)', Vacuum, 1987, 37, (8-9), pp.659-668

[5] Dayton, I.E., Shoemaker, F.C., and Mozley, R.F.: 'The measurement of two-dimensional fields. Part II: Study of a quadrupole magnet', Rev. Sci. Instrum., 1954, 25, pp.485-489

[6] Austin, W.E., Holme, A.E., and Leck, J.H.: 'The mass filter: Design and performance' (Chap. 10 in 'Quadrupole mass spectrometry and its applications', Elsevier, Amsterdam, 1976, pp.121-152)

[7] Ferran, J.: 'Residual gas analyser utilizing a miniature quadrupole array'. US Patent 5,401,962, 28 March 1995

[8] Schroeder, C.M.: 'Accurate silicon spacer chips for an optical fiber cable connector', Bell. Syst. Tech. J., 1977, 57, (1), pp.91-97

[9] Syms, R.R.A., Tate, T.J., Ahmad, M.M. and Taylor, S.: 'Fabrication of a microengineered quadrupole electrostatic lens', Electronics Letters, 1996 32, (22), pp. 2094-2095 